\title{
CONFLICTO, IDENTIFICACION Y PODER EN LA INFLUENCIA MINORITARIA
}

\author{
Gabriel Mugny* \\ Tomás Ibáñez** \\ Francisco Elejabarrieta** \\ Lupicinio Iniguez** \\ Juan A. Pérez* \\ * Universidad de Ginebra \\ ** Universidad Autónoma de Barcelona
}

\section{Resumen}

En 6 condiciones experimentales se presenta a los sujetos un texto minoritario extremamente favorable al aborto. siendo evaluada la influencia en una dimensión directa (actitud frente al aborto) y en una dimensión indirecta (actitud frente a la contracepción). En tres condiciones se introduce una pequeña amenaza y en las otras tres una fuerte amenaza que implica un fuerte costo social simbólico en caso de un eventual acuerdo con la minoría. En cada caso, o bien no se explica ninguna identificación (condiciones control) o bien se lleva a los sujetos a percibirse identificados ya a la Iglesia ya a la minoría. Los resultados principales muestran que a) en las condiciones sin identificación explícita, la minoría obtiene el efecto de conversión esperado por el modelo de la influencia minoritaria, en el caso de que el costo social es elevado; b) por el contrario, en caso de identificación explícita con la minoría el costo social disminuye tanto la influencia indirecta como la directa, lo que aboga en defensa del modelo del poder; c) en las condiciones en las que los sujetos son identificados con la Iglesia aparece un curioso efecto: cuanto mayor es el costo social más se acercan los sujetos a la minoría. Dado que las explicaciones divergen según la interpretación que los autores dan de este efecto paradójico y del conjunto de los resultados, se presentan dos interpretaciones complementarias, incluso aunque se contradigan entre ellas.

\section{Abstract}

An experiment on minority influence was designed with six experimental conditions. Three conditions imply an important threat and the others a little one: In each case an iden-

Este trabajo ha sido realizado en parte en el marco del proyecto patrocinado por el Fondo Nacional Suizo de Invesrigación Científica n? 1.714.0.83. 
tification with the Church or the minority is induced in the subjects or there is not identification at all. Results show that: a) in conditions without explicit identification, minority reaches the conversion effect if the cost is high, b) in the cases with explicit identification with the minority direct and indirect influence declines, c) when there are an identification with the Church there is a curious effect: higher is the cost more important is the minority effect. Two complementary interpretations of results are given.

\section{Introducción}

Desde que Moscovici planteara el tema en los años sesenta (cfr. Faucheux y Moscovici, 1967), el interés por el estudio de la Influencia Minoritaria no ha dejado de ir creciendo en el seno de la Psicología Social europea y ha introducido incluso alguna cuña en el ámbito de la norteamericana (cfr. Moscovici, Mugny y Van Avermaet, 1985).

No es exagerado decir que en el espacio de unos veinte años el aparato teórico elaborado en torno a la Influencia Minoritaria, las investigaciones que ha inspirado y los datos empíricos que ha generado, han configurado un auténtico programa de investigación, en el que, por analogía a la terminología lakatiana (cfr. Lakatos 1983) se podría detectar un núcleo duro, un cinrurón protector y sus heuristicas positivas y negativas. De hecho este programa de investigación ha tenido una incidencia sobre el planteamiento mismo de la temática de la influencia social en su acepción más general, evidenciando los límites y las caracrerísticas, tanto epistemológicas (mecanicismo) como sociales (mantenimiento del status quo) del enfoque clásico. señalando sus lagunas (imposibilidad de explicar el cambio social) y reinterpretando sus planteamientos y sus datos (por ejemplo, inversión teórica de la interpretación del experimento de Asch, 1951. sobre el conformismo).

$\mathrm{El}$ «núcleo duro» del programa de investigación sobre Influencia Minoritaria puede ser considerado, hoy por hoy, sólidamente establecido:

a) Contrariamente a lo que postulan las teorías funcionalistas de la dependencia, se ha demostrado que una fuente puede ejercer una influencia aunque no disponga del poder que confiere el número (mayoría), del prestigio que da el reconocimiento (competencia) o de la fuerza que proporciona el recurso a dispositivos de sanciones (poder, en el sentido pre-foucaultiano del término; cfr. Cartwright, 1959).

b) Las condiciones de posibilidad de dicha influencia pasan por la posesión de una contra norma claramente expresada (anclaje nómico que indica un punto de acuerdo posible con la fuente), y por la defensa consistente de esa contra norma (activismo que se traduce en el estilo de comportamiento de la fuente y que indica su voluntad de no ceder, así como su seguridad en sí misma).

c) El mecanismo de dicha influencia se basa en la creación de un conflicto de orden cognitivo y de orden social que conduce, por una parte, a una negociación más o menos implicita entre los protagonistas de la situación de influencia y, por otra parte, a una reconsideración del objeto de creencia cuestionado por la fuente.

A partir de aquí (Moscovici, 1976), decenas de investigaciones, de tipo básicamente experimental, han ido perfilando las distintas variables que inciden sobre la posibilidad y el grado de eficacia de la Influencia Minoritaria. Enumeremos algunas:

-Efectos de las defecciones que surgen en la mayoría y recíprocamente (Kiesler y $\mathrm{Pa}$ llak, 1975), efectos de apoyo social que puede recibir el representante de la mayoría (cfr. Doms y Van Avermaet, 1985).

-Diferencia entre las minorías pro-normativas (integrismo, ortodoxia,...) y las minorías anti-normativas (disidencia, heterodoxia,...) (vgr.: Deconchy. 1985; Mugny, 1975; Paicheler, 1977). 1983).

-Efectos de la psicologización y de la sociologización de la minoría (vgr.: Papastamou, 
- Diferencia entre estilo de comportamiento consistente pero rígido y consistente pero flexible (cfr. Mugny, 1981).

- Efectos debidos a la importancia numérica de la minoría (vgr.: Nemeth, Wachrler !. Endicott. 1977).

-Diferencias entre minorías intragrupo y extragrupo (vgr.: Pérez y Mugny, 1985a).

- Efectos de los contextos normativos (de originalidad frente a un contexto de objerividad. etc. (cfr. Mugny y Papastamou, 1984).

En una perspecriva más fundamental hay que destacar dos desarrollos que se han originado a partir de los planteamientos realizados en el equipo de la Universidad de Ginebra que trabaja sobre Influencia Minoritaria.

El primero consiste en una mayor coloración social de los procesos de Influencia Minoritaria. En efecto. si la intensidad del trabajo congnitivo desarrollado por el blanco de influencia para reposicionar el objeto, por lo menos a nivel privado, sigue siendo el motor del cambio de creencia, la intensidad del conflicto ya no se sitúa en un plano puramente cognitivo sino que está relacionada con los procesos grupales de redefinición de la identidad social del blanco de influencia (Mugny, Kaiser, Papastamou y Pérez, 1984). Adoptar el punto de vista minoritario implica también una identificación con la minoría y especialmente con sus atriburos más resaltados en la situación de influencia. El conflicto tiene pues una fundamentación social innegable y conviene captarlo en su pleno entramado social.

El segundo desarrollo consiste en la puesta en evidencia del complejo entramado que pone en relación la intensidad del conflicto, por una parte, y los tipos de resoluciones de este conflicto que se traducirán en una influencia captable a un nivel más o menos directo. Conflicto e influencia no se relacionan siguiendo una función lineal. La relación que se establece entre la intensidad del conflicto y la tasa de influencia tiende a configurar una curva en $U$ inverrida (Mugny y Pérez, en prensa). En efecto, existe un punto óptico de conflicto a partir del cual la influencia ejercida deja de intensificarse y empieza a decaer. El coste simbólico de la identificación con la minoría se torna excesivo y los mecanismos de resistencia a la influencia se movilizan con eficacia. Por lo menos aparentemente.

En efecto. las cosas no son tan simples y a la intensidad del conflicto y al coste simbólico de la identificación hay que adjuntarles otros procesos dinámicos que se escenifican en las situaciones de influencia minoritaria. Estas están colocadas también por modificaciones indirecras de las creencias del sujetn, unas modificaciones más profundas, más alejadas del tema abordado por la fuente minoritaria. En definitiva, un proceso de conversión según la expresión del propio Moscovici (1980). Estos desarrollos constituyen un notable enriquecimiento del «paradima» de la Influencia Minoritaria, arraigando en la realidad social y recogiendolo en su complejidad constitutiva.

Hay que reconocer que en la mayoría de los trabajos anteriormente señalados el costo de la identificación con la minoría es puramente simbólico e implícito y únicamente ha constituido una mera representación mental supuestamente elaborada por el propio sujeto. ¿Qué pasa cuando se introduce explícitamente en la situación de influencia minoritaria el coste social implicado por la disidencia y el dispositivo de sanción de que dispone todá institución social (Ibáñez, 1983)? En definitiva, ¿qué ocurre cuando se hace resaltar la red de relaciones de poder en que en realidad se desenvuelve toda situación social? Es la común preocupación por esta problemática la que condujo al planteamiento de esta investigación conjunta entre la Universidad de Ginebra y la Universidad Autónoma de Barcelona.

En esta investigación trataremos de sintetizar la problemática en torno a dos aspectos (variables independientes) que trataremos de poner a prueba experimental. Por una parte, se acentuará la oposición normativa que enfrenta las posiciones ideológicas de la minoría y del «poder» (concretamente, representado en la Iglesia). Este carácter general de oposición será acompañado de un grado variable de sanción que el «poder» explicita en caso de aceptarse las posiciones normativas que van contra él (concretamente, la posición defendida por 
la minoría). Asi pues, se inducirá explícitamente un grado variable (alto o bajo) del costo social que conlleva la aceptación del punto de vista minoritario.

En segundo lugar, el plan experimental cruzará la anterior variable independiente con la manipulación de la identificación del sujeto blanco de influencia con la Iglesia o con la minoría. Existen por supuesto dos grupos de control (alto o bajo costo social) en los que no se explicita ninguna identificación.

Las mediciones de la influencia se efectuarán tanto a nivel directo (actitud directamente relacionada con la postura defendida en el mensaje minoritario) como a nivel indirecto (accitud frente a un tema no mencionado en el mensaje).

Las hipóresis formuladas hacen referencia a dos contextos teóricos distintos. Centrémonos en primer lugar en el costo social que se explicita en la siruación de influencia. Partien. do del contexto del "paradigma» de las Relaciones de Poder se puede esperar que cuanto mayor sea el coste social de la adopción del punto de vista minoritario, menor será la influencia ejercida por éste. Simplemente porque en el caso de alto costo social se explicita más las sanciones y amenazas impartidas por el poder contra los eventuales disidentes.

Por el contrario, en el contexto del «paradigma» de la Influencia Minoritaria la inducción de tal costo social puede adquirir el significado de una acentuación del conflicto que opone la norma minoritaria a la norma defendida por el poder, en cuyo caso el alto costo social acentuaría más el conflicto que la inducción del bajo costo social. En este caso se espera que cuanto más intenso sea el conflicro inducido mayor será el cambio de opinión provocado en los sujeros experimentales.

En cuanto al grado de identificación del sujeto con cada una de las categorías sociales perrinentes, el modelo del Poder predice que cuanto más identificado esté el sujeto con el poder más estarán reguladas sus opiniones por él. Concretamente: los sujetos que son llevados a identificarse explícitamente con el poder deberán ser menos influenciados por la minoría (o más por el poder). Finalmente, según este modelo no se esperan diferencias entre influencia directa e indirecta.

En términos del modelo de la Influencia Minoritaria esto significa que cuando el sujeto explicita su acuerdo con la fuente minoritaria no sólo se autoatribuye la posición ideológica de la minoría, sino que además se autoabtribuirá otras características o atributos de la minoría resaltados en la situación de influencia (Mugny, Kaiser y Papastamou, 1983). Puesto que el costo social hará resaltar más los atributos negativos de la minoría, se entiende que tal autoabribución podría desembocar en una identificación psicosocial costosa, que será tanto más evitada cuanto mayor sea la identificación (inducida experimentalmente) con la minoría.

Ahora bien, en caso de escenificarse tal conflicto de identificación, la influencia no sería necesariamente nula sino que, por el hecho mismo del conflicto (de alguna manera interiorizado, por el simple hecho de no haber podido ser resuelto a nivel directo), se puede «desplazar» la resolución de este conflicto a niveles más indirectos (cfr. Moscovici, 1980).

Veamos ahora en deralle el experimento diseñado para confrontar esta serie de hipótesis, a veces opuestas.

\section{Método}

Sujetos

270 escolares de ambos sexos (154 mujeres y 116 hombres) y de edades comprendidas entre 15 y 19 años (mediana de 16 años), han participado en este experimento que se llevó a cabo en un Instituto de Enseñanza Media situado en una zona céntrica de Barcelona. El experimento se desarrolló en los locales y horas que habitualmente los alumnos frecuentaban para asistir a sus clases cotidianas. 


\section{Procedimiento experimental}

Se inciaba el experimento indicando a los sujetos que en el marco de un programa general de estudios sobre las opiniones de la gente frente a diversos temas de actualidad. nos interesábamos en ese momento concreto en el tema del aborto. Tras recoger algunos datos individuales (edad, sexo y curso), los sujetos comenzaban emitiendo (en el anonimato total) sus opiniones frente al aborto a través de cinco items concebidos para tal propósito (pretest). Inmediatamente después sucedían las inducciones experimentales. En primer lugar se inducía la identificación con una entidad social (con «los cristianos opuestos al abor10» 0 «un grupo minoritario favorable al aborto»). Para ello, los sujetos debían señalar una serie de características que plausiblemente en base a un estudio sociológico tendrían en común con una de esas entidades sociales. Así pues, legitimada por un supuesto estudio sociológico, se creaba de este modo una identidad de hecho entre el sujeto y una entidad social totalmente opuesta al aborto o con un grupo totalmente a favor del aborto. Esta identificación era controlada por una situación experimental en la que los sujetos no eran llevados a explicitar ninguna identificación (condición control).

Se manipulaba a continuación el grado de costo social de una actitud totalmente favorable al aborto. Para ello a todos los sujetos se les mencionaba la oposición de la lglesia al aborto en general. Ahora bien, mientras que en unas condiciones se les decía únicamente que «la Iglesia está totalmente en desacuerdo con el aborto», en las otras se acentuaba el grado del costo social simbólico: se decía que «la Ig lesia condena el aborto como un crimen y considera que los que están a favor son inmorales, irresponsables y egoistas».

A continuación venía la fase de la influencia: todos los sujetos eran sometidos a una influencia minoritaria. Se les presentaba para esto un texto en el que se defendía una posición totalmente a favor de la legalización y gratuidad del aborto. Para la totalidad de los sujetos este texto era atribuido a un grupo minoritario de jóvenes. Se medía a continuación la representación que los sujetos elaboraban de la fuente minoritaria. Los sujetos terminaban expresando sus opiniones (medida de influencia) frente al aborto y los anticonceptivos sobre un cuestionario de 25 items (que describiremos más adelante).

Veamos ahora una breve descripción del material experimental empleado cuyos detalles pueden verse en Pérez. y Mugny (1958b).

\section{El pretest}

Los sujetos comenzaban expresando sus posiciones frente al aborto. Para ello debían contestar (a través de una escala de tipo Likert en 7 puntos: $1=$ totalmente en desacuerdo; $7=$ totalmente de acuerdo a los cinco items siguientes:

- «hay que legalizar el aborto»;

- «las menores de edad también deben poder abortar»;

- «legalizar el aborto es una exigencia democrática»;

- «el aborto tiene que ser gratuito para todo el mundon;

- «siempre que una mujer ha quedado embarazada por violación hay que poder aplicarle el aborrom.

Las respuestas a este cuestionario nos servirán para tres objetivos:

a) En primer lugar para controlar eventuales diferencias de las opiniones entre las diferentes condiciones experimentales, que en principio no nos esperamos, dada la distribución aleatoria de los sujetos en las diversas condiciones experimentales.

b) Sobre la media de las respuestas a estos cinco items, separaremos aquellos sujetos que tienen opiniones menos favorables al aborto, y que por lo tanto están más opuestos de entrada al contenido del mensaje minoricario, de los sujetos con opiniones más favorables al aborto. Por comodidad del lenguaje y con respecto a la posición de la fuente minoritaria, 
nos referiremos a los primeros como «sujetos alejados» y a los segundos como «sujetos próximos».

c) Por último. las respuestas a este cuestionario serán consideradas como el punto de anclaje de las opiniones de los sujetos antes de recibir el mensaje. De este modo, una vez realizada la normalización correspondiente de las escalas, se procederá a calcular el índice del cambio de opiniones que estará constituido por la diferencia entre las opiniones a este cuestionario y las manifestadas por los sujetos después de haber pasado la fase de influencia propiamente dicha.

\section{El texto}

En la fase experimental de influencia propiamente dicha, los sujetos debían leer un texto atribuido a un grupo minoritario de jóvenes en el que se defiende una posición claramente a favor del aborto. Este texto se organiza en torno a la dimensión de la legalización y de la gratuidad del aborto. En cada una de estas dos dimensiones se presentan dos reivindicaciones: por una parte, se subraya la necesidad absoluta de manifestar públicamente la exigencia de legalizar totalmente el aborto y la elaboración de un estatuto jurídico que permita recurrir al aborto; por otra parte, se reivindica la gratuidad total del aborto y que la Scguridad Social debe asumir todos los costos.

Al final del texto se reiteran las dos reivindicaciones centrales: «en sintesis, nuestro grupo minoritario de jóvenes reivindica la legalización total del aborto y que éste sea totalmente gratuito para loda la génte».

Por último, el estilo del texto sistemáticamente remite a expresiones que subrayan el carácter indispensable de las reivindicaciones, lo que debe transferir un carácter conflictual específico a la posición en él defendida.

\section{El cuestionario de la imagen de la minoría}

Los sujetos debían juzgar la fuente minoritaria a través de una treintena de escalas bi. polares de 7 puntos. Semejante a un diferencial semántico, se introdujeron varias dimensio. nes. Así. se encuentra la dimensión de la consistencia (coherente-incoherente, seguro de sí miso-no seguro de sí mismo) de la tolerancia (rolerante-intolerante, tansigente-intransigente, flexible-rígido), de la representación social (portavoz-no representa a nadie, convincente-no convincente). de la credibilidad (digna de fe-no creíble, competente-incomperente), de la moralidad (moral-inmoral, egoísta-generoso), de la autonomía (partidista-autónomo. independiente-dependiente, individualista-sectarista). El resto de los items que componen este cuestionrio son: humanitario-político, reaccionario-progresista, viejo-joven. maduroinmaduro, mayoritario-minoritario, marginal-integrado, democrático-autoritario, feministaantifeminista, realista-irrealista. atrevido-no atrevido, crítico-acrítico, abierto-lleno de prejuicios y objetivo-no objetivo.

\section{El cuestionario de opiniones}

Los sujetos debían expresar su grado de acuerdo o de desacuerdo sobre una escala de intensidad tipo Likert en 7 puntos ( $1=$ totalmente en desacuerdo, $7=$ totalmente de acuerdo) sobre un total de 25 items organizados en torno a dos dimensiones. Una hace referencia explícita a la legalización y gratuidad del aborto; la llamaremos escala directa. La otra, compuesta de los items referidos a los anticonceptivos, y por lo tanto sin ninguna referencia a las reivindicaciones del texto minoritario, la llamaremos escala indirecta. Estas dos escalas (para mayor detalle véase Pérez y Mugny, 1985b) no sólo reenvían a contenidos diferentes en sí, sino al hecho de haber constituido o no el objeto del contenido del texto minoritario 
en un caso y no en otro. El contenido de estas escalas será explícitamente definicional de la fuente minoritaria (escala directa) o no definicional (escala indirecta).

La escala directa está compuesta por los 18 items referidos al tema del aborto (medida de la influencia directa) y la escala indirecta por 7 items referidos a los anticonceptivos (medida de la influencia indirecta). El análisis de la influencia sobre estas escalas será realizado sobre la media de los items que componen cada una de esas dimensiones que serán normalizados y se les sustraerá la escala del pretest. Un signo positivo indicará que los sujetos cambian más de opiniones en la misma dirección de la fuente minoritaria autora del texto. Es decir, un signo positivo indica la influencia efectiva buscada por el grupo minoritario.

\section{Diseño y manipulaciones experimentales}

En un plan factorial $2 \times 3$ se han manipulado dos variables independientes: por una parte, el grado del costo social (bajo versus alto) que conllevaría la aceptación de las posiciones minoritarias y, por otra parte, la identificación del sujeto con diversas entidades sociales (un grupo de cristianos opuestos al aborto, un grupo minoritario favorable al aborto o ninguna explicitación de una identificación, correspondiendo esta última a una condición control).

Para la manipulación de la identificación, inmediatamente después de haber contestado al pretest, y por lo tanto antes de presentar a los sujetos el texto minoritario, se indicaba a los sujetos que «en base a una encuesta sociológica se habían encontrado una serie de características generales que eran comunes a los alumnos del instituto en el que estudiaban y un grupo minoritario que está extremamente a favor del aborto» (para la condición «identificación con la Iglesia», lo subrayado era sustituido por "los cristianos que están extremamente apuestos al aborton). Se les presentaba a continuación las seis características siguientes, supuestamente comunes a los sujetos y a uno de esos grupos: «mismo medio social», "mismo tipo de educación», «mismo tipo de familia», mismo tipo de simpatías políticas», «mismo tipo de actividad profesional» y «mismo tipo de educación religiosa». Entre estas seis características, se indicaba a los sujetos que, efectivamente, siempre en base a la supuesta encuesta sociológica, cinco de ellas eran comunes a él y al grupo de cristianos o al grupo minoritario (según la condición experimental). Se les pedía que señalasen estas cinco características. Como puede apreciarse, estas características reenvían todas ellas a aspectos sociológicos. Se le presentaban además a los sujetos otras 5 características de orden más «actitudinal»: «mismo tipo de sensibilidad», «mismo sistema de valores», «mismo grado de sociabili. dad», «mismo tipo de personalidad» y «mismo nivel intelectual». Entre estas cinco, también se pedía a los sujetos que eligiesen las cuatro características que más probablemente creían que la encuesta sociológica había puesto en común entre ellos y el grupo invocado.

Así pues, los sujetos eran llevados a realizar este «ejercicio de identificación» sea con los cristianos totalmente opuestos al aborto (que referiremos como condición de identificación con la Iglesia), sea con un grupo minoritario totalmente favorable al aborto (condición identificación con la minoría). En una condición control los sujetos no realizaban esta tarea de identificación explícita.

La otra variable independiente, referida al costo social de la adopción de posiciones favorables al aborto, se manipulaba una vez que los sujetos habían realizado (en las condi. ciones precisas) el «ejercicio de identificación» y también antes de que los sujetos leyeran el texto minoritario. Se manipularon dos grados de costo social: uno más elevado y otro menos.

En las condiciones de identificación se recordaba en primer lugar a los sujetos la tarea que acababan de realizar y se les anticipaba también que iban a ser expuestos a una posición minoritaria sobre la que tenían que expresar después sus propias posiciones. Evidentemente, a los grupos control, que no habían realizado la «tarea de identificación», no se les recor- 
daba dicha tarea. La consigna exacta era: «Ahora que ya has indicado las características que tienes en común con los "cristianos opuestos al aborto" ("el grupo minoritario favorable al aborto", para la otra condición) pasarás a leer un texto de un grupo minoritario en el que se defiende una posición favorable al aborro. A continuación deberás indicar en qué grado estás de acuerdo o en desacuerdo con su contenidon. Para los grupos control de la identificación explícita este texto comenzaba: «Ahora pasarás a leer...».

Para la condición de "bajo costo social" se adjuntaba:

«Antes de que pases a leer el texto te recordamos que la Iglesia está totalmente en desacuerdo con el abortos. De hecho sólo se recuerda la posición de la Iglesia que, como puede suponerse. es ampliamente conocida por todos.

Por contraste, en la condición de "alto costo social» se decía: «Antes de que pases a leer el texto te recordamos que la Iglesia condena el aborto como un crimen y considera que los que están a favor son inmorales, irresponsables y egoístas». En esta condición, la expre. sión del acuerdo con la fuente implica un costo social simbólico particularmente marcado puesto que revela una serie de características específicas poco gratificadoras para la identidad personal.

En resumen, se tienen que los sujetos eran llevados a identificarse sea con la Iglesia, sea con el grupo minoritario o con ninguno de los dos. A continuación se induce un costo social en caso de manifestarse de acuerdo con el aborto y, por inferencia, con el texto que habrán de leer a continuación. Este costo es de orden moral y simbólico y para la mitad de los sujetos era muy alto por contraste con la otra condición que referiremos como bajo costo social.

\section{Resultados}

\section{Verificación de la manipulación de las inducciones}

Como se recordará, para inducir una identificación de los sujetos con diferentes grupos se les pedía que extrapolasen 5 de entre 6 características de tipo más sociológico que según un supuesto sondeo de opinión los sujetos tendrían en común con el grupo pertinente. En efecto, de las 5 pedidas se obtiene una media global de 4.77. Esto indica que los sujetos siguen ampliamente la consigna experimental. Nos podemos preguntar si los sujetos aceptan más identificarse con el grupo de cristianos opuesto al aborto o con el grupo minoritario favorable al aborto. La media de respuestas indica que no hay ninguna diferencia $(\mathrm{m}=4.78$ para la condición con la Iglesia y $\mathrm{m}=4.75$ para el grupo minoritario). Luego los sujetos aceptan tanto un tipo de inducción de la identificación con un grupo como con otro. De igual modo, no hay diferencias en función de la variable costo social. Los sujetos que participaron en la condición de bajo costo social eligen una media de 4.82 características y los de la condición de alto costo social de 4.71. En realidad esto es lógico, puesto que la inducción de esta variable era posterior a la de la identificación.

La misma lógica se observa en las características de tipo más actitudinal. De las 4 pedidas (entre 5 posibles) se obtiene una media global de $3,80(\mathrm{~m}=3.80$ en la condición identificación con la Iglesia y $\mathrm{m}=3.80$ para la condición con el grupo minoritario). De igual modo, tampoco hay diferencias entre el bajo costo social $(m=3.80)$ y el alto costo social $(m=$ 3.82). Así pues, por el momento podemos estar seguros de que no existen asimetrías entre las diversas condiciones experimentales en el grado cuantitativo de características elegidas, lo que nos sitúa en condiciones ideales para examinar los resulados de influencia que se producen.

Sin embargo, antes conviene anotar todavía dos comentarios: a) entre las características sociológicas la menos elegida es el mismo tipo de «simpatías políticas» (elegida aproximada. mente por el $53 \%$ de los casos). Las más elegidas (aproximadamente el $93 \%$ de los casos) son el mismo tipo de «medio social», el mismo tipo de «educación» y el mismo tipo de «fa- 
miliar. Siguen a continuación las características misma «actividad profesional» y mismo tipo de «educación religiosa» (elegidas aproximadamente por el $70 \%$ de los casos). En el grado de preferencia de cada uno de estos contenidos de las características no se encuentra ninguna diferencia entre condiciones experimentales.

b) Entre las características «actitudinales», las más elegidas por los sujetos como comunes entre ellos y el grupo correspondiente invocado (aproximadamente por un $87 \%$ de los casos) son «el grado de sociabilidad», «el tipo de sensibilidad» y ael nivel intelectual». En último lugar se encuentra el mismo tipo de «personalidad» ( $57 \%$ de los casos) y «el sistema de valores» ( $61 \%$ de los casos). Sobre esta última se constata una diferencia entre el grupo de cristianos y el grupo minoritario. Así, los sujetos la eligen el $75,27 \%$ de las veces en común con el grupo minoritario, mientras que sólo la eligen el $46,51 \%$ de las veces en común con el grupo de cristianos. Se puede decir, pues, que sobre el sistema de valores los sujetos estarían más identificados con el grupo minoritario proabortista que con el grupo de cristianos antiabortista. Esto puede constituir una prueba de la tendencia global proabortista de la población y por lo tanto de que una identificación con la minoría sería más plausible que con la Iglesia.

\section{Influencia directa e indirecta}

Para comenzar el análisis de los resultados, examinemos en primer lugar las condiciones control en las que no se explicita ninguna identificación del sujeto ni con la Iglesia ni con el grupo minoritario. De hecho, estas condiciones pueden ser comparadas a una situación «clásica» de influencia minoritaria: los sujetos son expuestos al mensaje minoritario y a continuación expresan sus opiniones. No obstante, como se recordará, en una de estas dos condiciones la adopción de una posición favorable al aborto implicaría un costo social más elevado que en otra.

Según la teoría de la conversión en la influencia minoritaria (Moscovici, 1980), nos esperamos que la influencia aumentará con el incremento del conflicto, por lo que nos esperamos que en la condición de mayor costo social (mayor conflicto) la minoría deberá obtener mayor influencia. Este efecto se tiene que observar sobre todo en la escala indirecta (referida a los anticonceptivos).

Teniendo esto en cuenta, si observamos los datos del cuadro 1 , se constatan dos efectos: en primer lugar que a nivel directo no existen diferencias $(\mathrm{t} / 258=0,847$; n.s.) entre la influencia que produce la minoría en un contexto social en el que resalta poco el costo social $(m=+0,03)$ y cuando dicho costo social resalta más $(m=+0,12)$. Sin embargo, a nivel indirecto sí que se da la diferencia $(\mathrm{t} / 258=2,039 ; \mathrm{p}<0,042)$ y en el sentido habitual de los resultados de influencia minoritaria: cuando se acentúa el costo social los sujetos aceptan. más a nivel indirecto las posiciones que por inferencia corresponden a la minoría $(m=+0,30)$ que si dicho costo social es menor $(m=-0,09)$. Así pues, a través de la manipulación del costo social se pone aquí en evidencia el efecto indirecto que más suele caracterizar la influencia de las minorías.

Para continuar la exposición de los restantes resultados, retengamos que este resultado visto constituye ya una verificación de que la operacionalización del costo social ha sido efectiva, al menos desde el punto de vista de la influencia minoritaria. Es decir, la condición que referimos como inductora de un mayor costo social, acentúa el conflicto. Teniendo esto presente, si ahora examinamos el efecto del costo social en las condiciones en las que se acentuó la proximidad psicológica del sujeto con la fuente, es decir, en las que el costo simbólico de una identificación con la posición minoritaria resalta ampliamente, se tiene una inversión del efecto visto anteriormente. En efecto, a nivel directo (véase cuadro 1) los sujetos aceptan más la minoría con la que se les llevó a identificarse cuando el costo social es bajo $(m=+0,10)$ que cuando aumenta el costo social $(m=-0,21 ; t / 258=2,076 ; p<0,039)$. A 
nivel indirecto se encuentra un resultado parecido $(\mathrm{m}=+0,12)$ para la condición de bajo costo social y $m=-0,34$ para la condición de alto costo social), aunque estadísticamente poco significativo $(\mathrm{t} / 258=1,443 ; \mathrm{p}<0,149)$. Se tiene pues, aquí, una verificación de que el costo social puede obstaculizar la influencia que obrendrá una minoría tal como predice el modelo del poder, sobre todo cuando la identificación con la fuente «amenazada» es resaltada.

Cuadro 1: puntuaciones medias del cambio de opiniones para cada condición experimental referidas al aborto (influencia directa), a los anticonceptivos (influencia indirecta) y a la adición de las dos (total). Un signo positivo indica un cambio de opiniones en el postest en la dirección de la fuente minoritaria (una actitud más favorable al aborto y/o a los anticonceptivos). $n$ entre paréntesis.

ABORTO

ANTICONCEPTIVOS

TOTAL

\begin{tabular}{|l|cc|cc|cc|}
\hline & \multicolumn{2}{|c|}{ costo social } & \multicolumn{2}{c|}{ costo social } & \multicolumn{2}{c|}{ costo social } \\
& bajo & alto & bajo & alto & bajo & alto \\
\hline \multirow{2}{*}{ Iglesia } & \multirow{2}{*}{0,11} & $+0,10$ & $-0,04$ & $+0,10$ & $-0,07$ & $+0,10$ \\
& & & & & $(45)$ & $(41)$ \\
control & $+0,03$ & $+0,12$ & $-0,09$ & $+0,30$ & $-0,03$ & $+0,21$ \\
& & & & & $(46)$ & $(45)$ \\
minoría & $+0,10$ & $-0,21$ & $+0,12$ & $-0,34$ & $+0,11$ & $-0,27$ \\
& & & & & $(44)$ & $(49)$ \\
& & & & & & \\
\hline
\end{tabular}

Si resumimos los resultados de estas dos condiciones y los ordenamos en función del grado de identificación y del conflicto inducido, encontramos que cuando no se ha explicitado una identificación con la fuente, al aumentar el conflicto, aumenta la influencia (sobre todo indirecta). Por el contrario, cuando el sujeto se encuentra explícitamente identificado con la minoría, al acentuar el conflicto la influencia disminuye drásticamente. Se encuentra. pues, una curva en $U$ invertida que da cuenta de la relación entre la influencia y el conflicto ya vista en otros trabajos (Mugny y Pérez, en prensa).

En función del grado de proximidad psicológica del sujeto con la fuente minoritaria, nos queda examinar separadamente lo que ocurre cuando los sujetos fueron identificados con la Iglesia, y por lo tanto cuando la identificación con la minoría fue menos explicitada. El primer resultado interesante (véase el cuadro 1) es que cuando los sujetos han sido llevados a identificarse con la Iglesia, y ésta «amenaza» fuertemente en caso de tomar posiciones proabortistas, los sujetos tienden a aceptar más las posiciones de la minoría a nivel directo $(\mathrm{m}=+0,10)$ que si dicha amenaza o costo social es menor $(\mathrm{m}=-0,11 ; \mathrm{t} / 258=1,669$; $\mathrm{p}<0,096$ ). Dicho de otro modo, cuando la Iglesia (con la que los sujetos por inferencia han sido identificados en esta condición) introduce una fuerte regulación en las posiciones que deban adoptar los sujetos, la influencia directa de este grupo Iglesia paradójicamente disminuye. Aunque estadísticamente no significativo $(\mathrm{t} / 258=0,031 ; \mathrm{n} . \mathrm{s}$.), a nivel indirecto se observa también la misma dirección de los resultados: la minoría obtendría también menos influencia en el contexto de bajo costo social $(\mathrm{m}=-0,04)$ que en el de alto costo social $(\mathrm{m}=$ $+0,10$ ).

Esto constituye una proceso paradójico del funcionamiento de la categorización (Pérez. 1985): la identificación con un grupo en un contexto de fuerte «efervescencia» social provoca 
que los sujetos rechacen, al menos en función de las posiciones ideológicas, más una identificación con dicho grupo. Se tiene así que en este contexto de alto costo social (representado además por la Iglesia) cuando los sujetos son llevados a identificarse con la Iglesia, en cuyo caso cabría esperar que las opiniones de éstos fuesen más reguladas por la Iglesia (tanto en función de la identificación como del costo social), y por lo tanto fuesen más opuestas al aborto, los sujetos manifiestan opiniones más favorables al grupo minoritario y, por contraste, menos a favor de la Iglesia. Los sujetos escapan de este modo a la fuerte regulación de las opiniones por el grupo Iglesia.

Esta misma paradoja de la categorización se encontró también, como vimos, cuando el sujeto fue explícitamente identificado con el grupo minoritario en el contexto de alta «efervescencia» social. En este caso rechazaban también más las posiciones minoritarias que cuando dicha identificación no fue tan explicitada o el costo social era inferior, contrariamente, pues, a lo que se podía esperar en función de la categorización (Tajfel, 1978) y diferenciación categorial (Doise, 1976).

Estos dos resultados que atestiguan las paradojas de la categorización, quedan avalados por la condición en la que no se explicita una identificación (ni con la Iglesia ni con la minoría) que, como ya vimos, en un contexto de fuerte conflicto era en el que se producía sobre todo una influencia indirecta.

Como ya hemos visto los resultados de influencia llevan la misma dirección tanto a nivel directo como a nivel indirecto, aunque no la misma intensidad, lo que tiene su significación como ya hemos comentado. Ahora, para resumir de otro modo más global los efectos, vamos a considerar las condiciones sobre las puntuaciones medias referidas a la escala del aborto y la de lós anticonceptivos adicionadas (véase el cuadro 1).

El conjunto de las condiciones puede ser ordenado teniendo en cuenta, en primer lugar, si el costo social es bajo o alto y, en segundo lugar, los tres grados de identificación con la minoría. La gráfica 1 resume los resultados de influencia de cada condición ordenados con esa lógica. Como puede apreciarse, se dibuja una curva en $U$ invertida (el test de «trendanalysis» arroja que la curva es cuadrática: $F(1,258)=9,256 ; p<0,003)$. El significado de esta curva es que cuando la tensión social es menos fuerte (introducida por medio del costo social, a través de un simple recuerdo de la posición de la Iglesia, de hecho ampliamente conocida) la influencia se produce linealmente en función de la identificación del sujeto con cada categoría social invocada. En primer lugar, si el nivel de identificación a la minoría fue explicitado (máximo grado de identificación con la minoría), ésta obtiene más influencia $(m=+0,11)$ que en las otras dos condiciones en las que el grado de identificación por comparación es menor $(m=-0,03$ para la condición control y $m=-0,07$ para la condición identificación con la Iglesia). Corolariamente, cuando se explicita la identificación con la Iglesia, la minoría obtiene menos influencia que en las otras dos condiciones. Así pues, la explicitación de la identificación con la Iglesia, en este contexto de bajo costo social, aumenta la resistencia a la influencia minoritaria, lo que podríamos decir que se traduce por un aumento de la «influencia» de la Iglesia. Para resumir, en un contexto de poca «efervescencias social, la identificación con la fuente minoritaria (instaurándose, pues, de este modo una situación intragrupal), aumenta la influencia de la minoría. Este resultado es totalmente coherente con otros ya existentes en la literarura (Mugny y Papastamou, 1982) que mostraron que una identificación de tal tipo provocaba efectivamente un aumento de la influencia de la minoría. 
Figura 1: representación gráfica (cfr. cuadro 1) del grado de influencia total (adición de la escala referida al aborto y a los anticonceptivos) correspondiente a cada condición experimental en función del costo social y del grado de identificación.

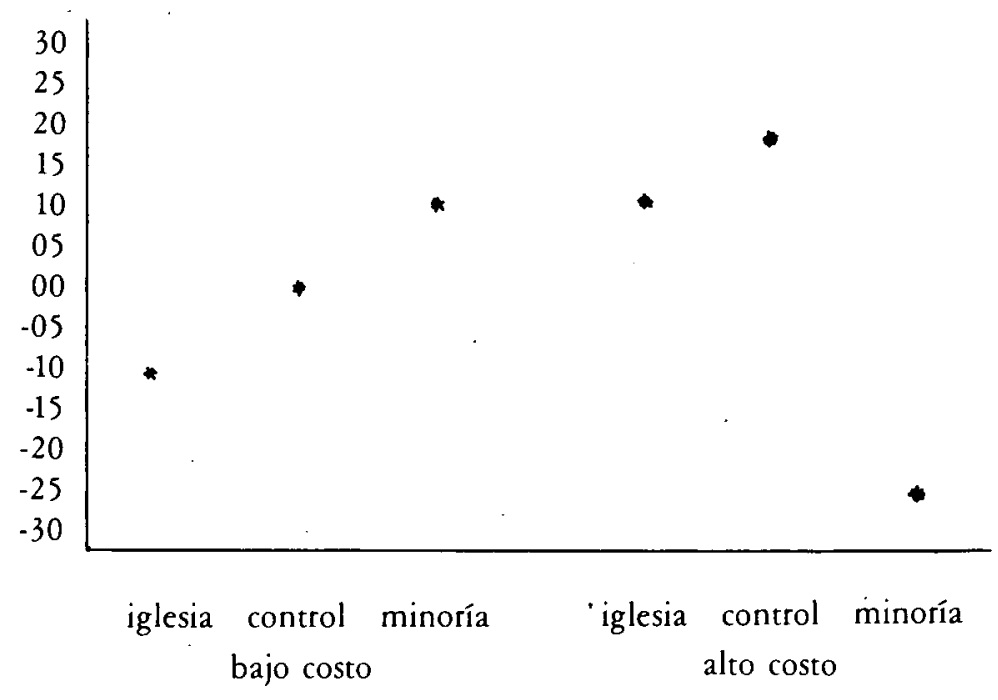

Por el contrario, cuando el costo social aumenta y pone en juego una identificación social conflictual, la influencia sigue una función inversa al grado de identificación de la fuente: cuando el sujeto se encuentra más identificado con la fuente minoritaria se obtiene menos influencia $(m=-0,27)$ que si el grado de identificación con la fuente no fue explicitado $(\mathrm{m}=+0,21)$ o fue identificado con la categoría que se opone a la minoría $(\mathrm{m}=+0,10)$.

Si resumimos los resultados en conjunto de las seis condiciones experimentales tenemos: a) cuando el conflicto es «intermedio» (bajo costo social) la identificación con la minoría aumenta su influencia. b) Cuando el conflicto aumenta (alto costo social), si no se explicitó una identificación con ninguna categoría social invocada, la influencia obtenida por la minoría aumenta. Cuando esta identificación se explicita se produce la paradoja de la categorización: los sujetos más identificados con la Iglesia ahora rechiazan más la regulación de las opiniones que introduce la Iglesia y los más identificados con la minoría también rechazan las posiciones ideológicas de la minoría.

\section{Análisis complementarios}

Dos tipos de análisis, uno referido a la representación elaborada por los sujetos de la fuente minoritaria y otro en función del sexo de los sujetos, nos aportan aún alguna información complementaria a los resultados vistos anteriormente.

\section{Representación de la fuente}

Respecto a la representación de la fuente retenemos únicamente aquellos factores (lamentablemente pocos) que arrojan algún efecto significativo. En efecto, el análisis factorial realizado sobre el cuestionario de la imagen de la fuente arroja nueve factores y únicamente haremos un comentaio de los cinco primeros, así pues, los más importantes. El primer factor refiere el aspecto evaluativo de la fuente y están saturados la mayor parte de los items. Este factor no arroja ningún efecto significativo entre las diferentes condiciones. 
Las primeras diferencias entre condiciones las encontramos en el segundo factor que representa la dimensión de la flexibilidad-rigidez (flexible, tolerante, transigente). En este se encuentra una verificación relativa de la efecrividad de la inducción del costo social. Así, cuando se indujo un alto costo social, la fuente minoritaria es juzgada como más rígida (o menos flexible) que cuando se indujo un menor costo social. Ahora bien, este efecto sólo es significativo $(\mathrm{t} / 258=1,982 ; \mathrm{p}<0,049)$ en las condiciones referidas a sidentificación con la minoría» y la condición acontrol». Es decir, en estas dos condiciones el costo social alto acentúa la percepción de la fuente como rígida, y por lo tanto testimonia un resentimiento más intenso del conflicto.

Sin embargo, como se recordará, a nivel de la influencia obtenida estas dos condiciones diferían ampliamente entre ellas. En la que se explicitó la identificación con la minoría no se obtenía una influencia positiva (de hecho, más bien un rechazo de las posiciones minoritarias), contrariamente a lo visto en la condición control en la que sí se liberaba una influencia. Ahora bien, globalmente se tiene que en la condición sin identificación explicitada, la minoría es también percibida como más autónoma e independiente y se la percibe como más feminista que en el resto de las condiciones. Se tendría así que en esta condición control la minoría es percibida como más portadora de una «alternativa socialı o con mayor arepresentatividad social». De hecho, este grado de representatividad social es más atribuido a la minoría en esta condición control cuando resaltó el alto costo social, que como vimos era la condición en la que se producía una mayor influencia (sobre todo a nivel indirecto).

Así pues, para resumir estos resultados de la representación de la fuente tenemos: a) en la condición sin identificación explícita se percibe a la minoría como más autónoma y más feminista que en las otras condiciones. En cierto modo, se la reconoce como más portadora de una alternativa social. Por otra parte, dentro de esta condición sin identificación, la acentuación del costo social conlleva que se perciba a la minoría como más rígida y con más representatividad social, lo que daría cuenta de la influencia indirecta que vimos en esta condición.

b) En la condición en la que los sujetos fueron identificados con la minoría, la inducción de un alto costo social conlleva también la atribución de un mayor grado de rigidez a la minoría. Pero al mismo tiempo, es en esta condición de identificación y alto costo social, en la que la fuente minoritaria no es percibida como feminista, lo que parece indicar que se trata de una negación de la minoría como entidad social alternativa. El resultado fue poca influencia (si no negativa).

A pesar de la prudencia necesaria con la que hay que interpretar estos resultados referidos a la representación de la fuente, no obstante, aportan algunas indicaciones sobre las significaciones que el costo social introduce. Estas significacionies parecen estar ampliamente relacionadas con las identificaciones introducidas o no.

\section{El efecto de la ridentidad" sexual de los sujetos}

El segundo tipo de resultado que arroja una información complementaria a los resultados de influencia que hemos visto, viene dado por la interacción entre la identidad sexual de los sujetos y la influencia. En realidad, esta interacción únicamente arroja un efecto en la condición en la que se llevó a los sujetos a identificarse con la minoría. Como se recordará, en esta condición, cuando se acentuaba el costo social, no sólo no se producía una influencia positiva de la fuente, sino incluso un relativo rechazo. La minoría no obtenía una influencia debido probablemente al fuerte conflicto de identificación escenificado en tal situación. En realidad, es cierto que también en esta condición podíamos habernos esperado una cierta influencia indirecta y este no fue el caso. 
Por otra parte, sabemos que el tema del aborto interpela más la identidad social femenina que la masculina (Pérez y Mugny, 1985b). De este modo, podemos suponer que la identificación con la minoría sería más pertinente para las mujeres que para los hombres. O dicho sea de otro modo, las mujeres se sentirían más identificadas con la minoría que los hombres. Siguiendo la lógica de los resultados que hemos visto, según la cual se rechazaba a la minoría cuando el costo social es alto y los sujetos se encontraban de lleno identificados con ella, se debería observar ahora que efectivamente este rechazo es más acentuado en el caso de las mujeres que de los hombres.

Al examinar la condición en la que los sujetos füeron llevados a identificarse con la fuente minoritaria en el contexto de alto costo social, en función del sexo de los sujetos (única condición en la que se produce un efecto significativo) se tiene que las mujeres rechazan más la fuente minoritaria a nivel indirecto $(m=-0.55)$ que a nivel directo $(m=-0.10)$. Por el contrario los hombres rechazan más la fuenre a nivel directo $(m=-0,37)$ que a nivel indirecto $(m=-0,02)$. Así pues, en las mujeres se produce más influencia directa que en los hombres. Esto se invierte a nivel indirecto y los hombres son más influenciados que las mujeres, o son más influenciados a nivel indirecto que a nivel directo, siendo lo contrario pa:a las mujeres. Parece surgir así que las mujeres a nivel directo estarían más interpeladas para defender las posiciones proabortistas que los hombres. La presión social de la identificación es más fuerte sobre ellas que sobre los hombres. Este grado diferencial de presión social es el que daría cuenta de un efecto más positivo (o menos negativo) a nivel público pero no a nivel privado en el caso de las mujeres (a modo de un tipo de respuesta de complacencia, según la clasificación de Kelman, 1958) y un mayor rechazo a nivel público que privado en el caso de los hombres.

\section{Discusión}

Los resultados del presente experimento confirman en parte las hipótesis generales que se deducen de cada uno de los dos paradigmas teóricos confrontados.

En primer lugar, en relación con los grupos control, se confirma la tesis de la influencia minoritaria según la cual cuando se incrementa la intensidad del conflicto (concretamente, alto costo social) se observa un incremento de la influencia de la fuente minoritaria que se manifiesta sobre la dimensión indirecta (contraceptivos).

En segundo lugar, se encuentra que no ocurre así en las situaciones en que se induce una identificación explícita con la minoría. En efecto, tanto la influencia directa (aborto) como la influencia indirecta (contraceptivos) se reducen drásticamente cuando el costo social es alto. Es en estos casos la tesis del poder la que recibe plena confirmación. A pesar de incrementar la intensidad del conflicto, un costo social alto disminuye la influencia (tanto indirecta como directa) como si el sujeto percibiese entonces que las amenazas le conciernen más directamente y resistiera con mayor vigor a la influencia.

Ahora bien, se puede notar que este resultado no es totalmente contradictorio con la teoría de la influencia minoritaria, ya que la evolución actual del modelo (Mugny y Pérez, en prensa) presupone que la relación entre la intensidad del conflicto y la influencia se designa tanto por una relación lineal como por puntos de inflexión. Es decir, globalmente, adopta la forma de una curva en $U$ invertida, como vimos en la introducción. Indudablemente, la explicitación de las relaciones de poder en las situaciones de influencia parecen constituir uno de los mayores factores que pueden dar cuenta de la inflexión de dicha curva y puede también contrarrestar hasta el efecto de conversión.

Otro resultado que cabe destacar es que en las condiciones en las que se indujo una identificación con la Iglesia la influencia directa como (tendencialmente) la influencia indirecta son mayores cuando el costo social es alto. Parece entonces que la inclusión explícita 
del sujeto en el grupo que detenta el poder (Iglesia) le permite aceptar la influencia del minoritario tanto más cuanto más arriesgada sea dicha aceptación.

La interpretación teórica de este resultado difiere según la posición teórica de los autores. En efecto, en función de la teoría del poder estos resultados sugieren una reformulación y planteamiento de ciertas hipótesis interesantes. Así, el cambio social no constituirá un efecto directamente producido por la minorías, sino que sería un proceso indirecto. La minoría aporta ciertamente el contenido novedoso, pero serían los sujetos pertenecientes a la mayoría quienes se hacen eco de la postura minoritaria y, al igual que un caballo de Troya, la extienden en el tejido social, amparados en la seguridad y en la tranquilidad que les confiere su estatus de sujetos «bienpensantes».

Si los sujetos de la mayoría actúan de esta forma sería porque la adopción de las posturas minoritarias no conlleva peligro alguno para ellos, no hay riesgos de discriminación y de represalias. Expresan los puntos de vista minoritarios porque no se les puede confundir con la minoría. Cuando este riesgo aparece, es decir, cuando las amenazas les conciernen directamente, ya que se les identifica con los disidentes, entonces se echan para atrás. Un judio puede permitirse algún chiste antisemita porque difícilmente se le tildará de antisemita y, sin embargo, con ello no deja de hacer propaganda antisemita.

Si este proceso de extensión indirecto, o «desde dentrom, de las innovaciones es cierto, entonces también se explicaría que las innovaciones socialmente radicales, de orden dos, para retomar un término de Watzlawick (1976), tengan tan poco éxito. En efecto, hemos visto que los miembros de la mayoría dejan de aceptar la postura minoritaria cuando ésta representa un coste social elevado y se sienten directamente amenazados (identificación con la minoría), con lo cual solamente aceptarán y propagarán las innovaciones que no les sitúen ipso facto en el grupo de los disidentes, es decir, en definitiva aquellas innovaciones que no representan un peligro radical para las instituciones y que pueden ser integradas a la larga con algunos reajustes.

Desde el punto de vista de la teoría de la Influencia Minoritaria estos resultados revelan dos tipos de procesos. En primer lugar, y de acuerdo con la teoría del Poder, este resultado completa la teoría de la conversión, puesto que efectivamente el impacto de las minorías es indirecto. De hecho, este efecto no puede sino ser indirecto, puesto que los miembros de la población son llevados a redefinir, de manera constructivista, los objetos sociales en cuestión. Y si lo hacen sobre una dimensión indireta, ello se debe a que la dimensión directa conlleva de modo explícito mayores implicaciones sociales, sean de orden más simbólico o más «real» (riesgo de sanción). Cuanto más resaltan esas implicaciones mayor será el costo social que conlleva la posición minoritaria en caso de eventuales identificaciones, siendo en este caso mayor el conflicto inducido por la minoría. Estos resultados muestran también que existen puntos de inflexión a partir de los cuales la influencia indirecta es bloqueada.

Dicho esto, otros resultados (vgr.: Pérez, 1985, experimento 10) muestran que esta curva en $U$ invertida que relaciona la tasa de influencia y el conflicto, puede aplicarse también en niveles de influencia más indirectos. Introduciendo dos niveles de influencia indireta (el habitual referido a la contracepción y una media del «Zeitgeist»), se pudo poner de manifiesto que cuanto más aumenta el conflicto más la influencia aparece a nivel indirecto, situándose siempre los puntos de inflexión de la curva a niveles más elevados de conflicto. Esto nos lleva a una relectura del conjunto de los resultados que nos permitirá desvelar una auténtica paradoja, al menos desde el punto de vista de la teoría de la influencia minoritaria.

Esta paradoja ha sido ya detectada al categorizar la minoria como extragrupo (Pérez, 1985): dicho tipo de categorización tiende a impedir la influencia directa, a modo de una discriminación intergrupal (Tajfel, 1978), pero libera de forma tecurrente una influencia indirecta o latente. En conjunto, los resultados que resaltan en este experimento confirman la existencia de tales efectos paradójicos. En primer lugar el efecto de la identificación al poder, encarnado aquí en la Iglesia: cuanto mayor es el costo social mayor es la influencia 
de la minoría. Una posible interpretación es que los sujetos en este caso han percibido la minoría como un extragrupo, como representante de una mayor alternativa, percepción facilitada, paradójicamente, a través de la identificación a la Iglesia y de la acentuación del costo social que ésta introduce. Por el contrario, cuando los sujetos no son identificados de modo explícito ni a la Iglesia ni a la minoría (condición control) y se acentúa el costo social en caso de una eventual identificación con la minoría (al aceptar sus posiciones) aparece una influencia, pero únicamente a nivel indirecto. A hora bien, esta influencia está relacionada con una categorización de la fuente minoritaria como feminista, tal y como lo hemos visto en los datos relativos a la representación de la fuente. La identificación explícita de los sujetos con la fuente minoritaria en ese caso de alto costo social, inhibe ese proceso de conversión, observándose también en la imagen de la fuente una inhibición del reconocimiento de la minoría como alternativa. El costo social no sería así un efecto directo del miedo de una sanción, sino que relevaría de la construcción misma de la representación de la minoría, siendo así más conflictual, pero impidiendo ser categorizada como alternativa.

Finalmente, mientras que en la perspectiva del modelo del Poder esta situación de alto costo social sería «más realista» y «más válida ecológicamente», esto no sería forzosamente el caso desde la perspectiva minoritaria: el mismo carácter minoritario de la fuente también puede conllevar como consecuencia que la población (los sujetos) no se sienta identificada a la minoría de entrada, lo que posibilitaría la percepción de dicha minoría como una categoría alternativa, conllevando esta percepción los efectos latentes paradójicos que se conocen.

Se puede constatar sin esfuerzo, el acuerdo no es perfecto. En función del modelo los resultados adquieren un significado u otro. Al jugar el juego, y respetando las reglas del método hipotético-deductivo, cada modelo aporta efectivamente su parte de razón, una centración propia. En nuestra opinión, constituye un caso ejemplar de lo que podría ser una colaboración (además internacional) entre equipos de investigación en el que dicha colaboración no se reduce meramente a una mútua confirmación que desemboque en un consenso. Todo lo contrario, dicha cooperación se ha establecido sobre un auténtico conflicto de centraciones a partir del cual cada uno de los eparadigmas» teóricos sale enriquecido y del cual un día puede ocurrir que surja una síntesis que los superará a ambos. He aquí por qué los autores pretenden seriamente teincidir!

\section{REFERENCIAS BIBLIOGRAFICAS}

Asch, S.E. Effects of group pressure upon the modification and distortion of judgment. In H. GUETZKOW (Ed.), Groups, leadership and men. Pittsburgh: Carnegie Press, 1951.

Cartwright, D. (Ed.). Studies in social power. Ann Arbor: Univ. of Michigan Press, 1959 (a). Desconchy, J.P. The paradox of ortbodox minorities: when orthodoxy infallibly fails. Cambridge: Cambridge Univ. Press-L.E.P.S., 1985.

Doise, W. L' articulation psychösociologique et les relations entre groupes. Bruxelles: A de Boeck, 1976.

Doms, M., \& Van Avermaet, E. Social support and minority influence: the innovation effect reconsidered. In S. Moscovici, G. Mugny, \& E. Van Avermaet (Eds.), Perspectives on minority influence. Cambridge: Cambridge University Press-L.E.P.S., 1985.

Faucheux, C., \& Moscovici, S. Le style de comportement d'une minorité et son influence sur les réponses d'une majorité. Bulletin du Centre d'Etudes et de Recherches Psychologiques, 1967, 16, 337-360.

Ibañez, T. Poder y libertad. Barcelona: Hora, 1983. 
Kiesler, C.A., \& Pallak, M.S. Minority influence: the effect of majority reactionaries and defectors, and minority and majority compromisers, upon majority opinion and attraction. European Joumal of Social Psychology, 1975, 5, 237-256.

Lakatos, I. La metodología de los programas de investigación cientifica. Madrid: Alianza, 1983. Moscovici, S. Social influence and social change. London: Academic Press, 1976.

Moscovici, S. Toward a theory of conversion behavior. In L. Berkowitz (Ed.), Advances in experimental social psychology (vol. 13). New York: Academic Press, 1980.

Moscovici, S., Mugny, G., \& Van Avermaet, E. (Eds.) Perspectives on minority influence. Cambridge, París: Cambridge University Press, Editions de la Maison des Sciences de l'Homme, 1985.

Mugny, G. Bedeutung der Konsistenz bei der Beeinflussung durch eine konkordante oder diskordante minderheitliche Kommunnikation bei sozialen Beurteilungsobjekten. Zeitschrift fur Sozialpsychologie, 1975, 6, 324-332.

Mugny, G. El poder de las minorías. Barcelona: Rol, 1981.

Mugny, G., \& Papastamou, S. Minority influence and psycho-social identity. European Joumal of Social Psychology, 1982, 12, 379-394.

Mugny, G., \& Papastamou, S. Les styles de comportement et leur représentation sociale. In S. Moscovici (Ed.), Psychologie sociale. París: Presses Unversitaires de France, 1984.

Mugny, G., Kaiser, C., \& Papastamou, S. Influence minoritaire, identification et relations entre groupes: Etude expérimentale autour d'une votation. Cabiers de Psychologie Sociale, 1983, 19, 1-30.

Mugny, G., Kaiser, C., Papastamou, S., \& Pérez, J.A. Intergroup relations, identification and social influence. British Journal of Social Psychology, 1984, 23, 317-322.

Mugny, G., Pérez, J.A. Kaiser, C., \& Papastamou, S. Influence monoritaire et relations entre groupes: l'importance du contenu du message et des styles de comportement. Revue Suisse de Psychologie, 1984, 43, 331-351.

Mugny, G., Pérez, J.A. Influence sociale, conflict et identification. En prensa.

Nemeth, C., Wachtler, J., \& Endicott, J. Increasing the size of a minority: Some gains and some losses. European Journal of Social Psychology, 1977, 7, 15-27.

Paicheler, G. Norms and attitude change II: the phenomenon of bipolarization. European Journal of Social Psychology, 1977, 7, 4-14.

Papastamou, S. Strategies of minority and majority influences. In W. Doise, \& S. Moscovici (Eds.), Current issues in european social psychology (Vol. I). Cambridge: Cambridge University Press-L.E.P.S., 1983.

Pérez, J.A. Influencia minoritaria y procesos intergrupales: el conflicto frente a la discriminación. Madrid: Tesis Doctoral, 1985.

Pérez, J.A., \& Mugny, G. Categorización e influencia minoritaria. Anuario de Psicología, 1985,32,

Pérez, J.A., \& Mugny, G. Influencia minoritaria sobre las opiniones frente al aborto y los anticonceptivos. Estudios de Psicología, 1985, 24,.

Tajfel, $H$. (Ed.) Differentiation between social groups: studies in the social psychology of intergroup relations. London: Academic Press, 1978.

Watzlawick, P. y otros. Cambio. Barcelona: Herder, 1976.

\section{AGRADECIMIENTOS}

Queremos agradecer la colaboración de Jordi Catlla, Concepción Celaya, Joan Comas, Miques Coménech, Quim Ferrandiz, Juan Muñoz, Francisco Péres, M.a José Pubill, Carles Ramírez, Toni Ruiz, Toni Serra, Gemma Tubert, Félix Vázquez y Joan Vidal, sin los que no hubiera sido posible la realización del experimento incluido en este trabajo. 